\title{
Frequency Restoration Reserve Control Scheme with Participation of Industrial Loads
}

\author{
Davide Fabozzi \\ Dept. of Chemical Engineering \\ Imperial College London \\ London, UK
}

\author{
Nina F. Thornhill \\ Dept. of Chemical Engineering \\ Imperial College London \\ London, UK
}

\author{
Bikash C. Pal \\ Dept. of Electrical Engineering \\ Imperial College London \\ London, UK
}

\begin{abstract}
In order to accommodate larger amounts of renewable energy resources, whose power output is inherently unpredictable, there is an increasing need for frequency control power reserves. Loads are already used to provide replacement reserves, i.e. the slowest kind of reserves, in several power systems. This paper proposes a control scheme for frequency restoration reserves with participation of industrial loads. Frequency restoration reserves are required to change their active power within a time frame of tens of seconds to tens of minutes in response to a regulation signal. Industrial loads in many cases already have the capacity and capability to participate in this service. A mapping of their process constraints to power and energy demand is proposed in order to integrate industrial loads in existing control schemes. The proposed control scheme has been implemented in a 74-bus test system. Dynamic simulations show that industrial loads can be successfully integrated into the power system as frequency restoration reserves.
\end{abstract}

Index Terms-automatic generation control, frequency restoration reserves, industrial loads, process systems, secondary frequency control

\section{INTRODUCTION}

Electricity is a special commodity: since economical, extensive and efficient electric energy storage is yet to be found, produced and consumed active power must be equal at any instant. If production exceeds consumption, this results in an increase of the system frequency, i.e. an accumulation of the excess energy in the form of kinetic energy of the rotating masses that are connected to the system. Conversely, kinetic energy may be borrowed from the system when consumed power exceeds produced power, in the form of a decrease of system frequency.

In modern electric power systems, system frequency is tightly controlled because electric machinery is designed to operate in a strict frequency band around the nominal frequency. Frequency control is one of the main ancillary services that one Transmission System Operator (TSO) has to guarantee. A comparison of frequency control ancillary services across different systems is beyond the scope of this paper. However, it is widely recognised that, although slightly different in their implementation, most frequency control reserves can be ascribed to three categories [2]: primary, secondary and tertiary.

The financial support from the Marie Curie FP7-IAPP project "Using realtime measurements for monitoring and management of power transmission dynamics for the Smart Grid - REAL-SMART"[1], Contract No: PIAP-GA2009-251304 is gratefully acknowledged.
Traditionally, power system operators have relied on generators and transmission equipment to provide power system control actions, while loads have been regarded as the uncontrollable part of the system. The increasing share of renewable energies, however, reduced the overall controllability of generation, as the output of renewable generators is only partly controllable and largely unpredictable: for instance, one cannot precisely schedule the production of a wind park, as this depends on the wind inflow. Frequency quality degradation in the last decade led TSOs to re-examine frequency control services (which, for large part, have been set up decades ago in very different system conditions) and enlarge the pool of available reserves, mainly by allowing participation of industrial processes. Indeed, regardless of the specific causes, any frequency incident can be attributed to insufficient reserves.

Since decades, power engineers have been advocating for achieving more controllability of loads [3] and some stated that responsive loads are "the most underutilized reliability resource available to the power system" [4]. Industrial automation vendors are paving the way towards automatic and faster participation of industrial loads in power system operation: this concept is sometimes referred to as "industrial smart grid" [5].

In this paper, a procedure is proposed to map several industrial process constraints into power and energy constraints, providing a measure of their capability to provide frequency restoration service with as few as five parameters. The traditional Automatic Generation Control (AGC) scheme has been modified in order to accommodate industrial loads participation. The proposed control scheme has been implemented in a 74-bus test system. Dynamic simulations show that industrial loads can be successfully integrated into the power system as frequency restoration reserves.

\section{OVERVIEW OF FREQUENCY CONTROL SERVICES}

The classification of frequency control services into primary, secondary and tertiary is somewhat standard across continental Europe. However, since services have been developed independently by various system operators with different names and characteristics, confusion might arise when comparing services from different systems (it happens that completely different services go under the same name in different systems). A recent report by the European Network of Transmission System Operators for Electricity (ENTSO-E), uses a different pan-European harmonised terminology [6], which is the one adopted in this paper: 
- Frequency Containment Reserve (FCR). The objective of FCRs is to stabilise frequency to an acceptable value within tens of seconds. FCRs act in a fast, automatic and decentralised way, usually through a speed governor that controls the active power production of participating units in response to a frequency deviation signal. FCRs roughly correspond to primary frequency control reserves across continental Europe.

- Frequency Restoration Reserve (FRR). FRRs restore the frequency to its nominal value in order to relieve the units participating in FCR service. The time frame of FRR action ranges from tens of seconds to tens of minutes. FRRs are controlled centrally through a regulation signal sent to the speed governor of participating units in response to frequency deviation and transfer imbalance (i.e. the difference between scheduled and actual power transfers between control areas). AGC is the standard implementation of FRR control for generators. In some systems, this service is also referred to as Load Frequency Control. FRRs roughly correspond to secondary reserves.

- Replacement Reserve (RR). RRs are the slowest reserves that are activated manually in response to a request from the control center in order to free up the units participating in FCR and FRR controls. RRs act in a time frame from tens of minutes to hours. RRs roughly correspond to tertiary reserves.

The requirement for FCR is very time-critical. Most industrial loads are not likely to be able to provide this service, other than by the inertial response of their rotating machinery. On the other hand, loads such as industrial batch processes, metal processing plants and paper mills are already utilized across many systems as RRs [7]. Their use as FRRs is limited to one case: an experimental use of an aluminium smelter to provide FRR service has been conducted by Alcoa Inc. and Oak Ridge National Laboratory in the Midwest ISO system [8].

\section{AGC WITH PARTICIPATION OF INDUSTRIAL LOADS}

This paper proposes the use of industrial loads as FRRs, along with thermal and hydro generators, which are presently the preferred choice for this service. Several reasons suggest that industrial processes might represent a valid FRR resource. For instance:

- each industrial load accounts for a large consumption, which may be as high as tens or hundreds of thousands of domestic loads (it is obviously easier to control and communicate with a few large loads than with millions of domestic customers, as it is proposed by traditional "Smart Grid" approaches);

- industrial plants are supervised by trained personnel and have already installed some of the infrastructure needed for control and communication (e.g. power management systems, telemetry equipment);

- some electric loads inside a plant may contribute less to production and could therefore be temporarily switched off with few consequences on the productive process;

- additional FRR revenue may be unlocked with minimal or no new investment.
While the actual implementations may not be exactly the same in different systems, the AGC scheme described hereafter can be considered as a standard FRR service [9]. The AGC is based on the so-called Area Control Error (ACE). For a multi-area system, the ACE of area $a$ at time $t$ is defined as:

$$
A C E_{a}(t)=\lambda \Delta f(t)+\Delta P_{a}(t)
$$

where $\lambda$ is a frequency bias constant, $\Delta f(t)=f(t)-f_{N}$ is the deviation of the actual frequency $f(t)$ from the nominal $f_{N}$ and $\Delta P_{a}(t)=P_{a}(t)-P_{a}^{0}$ is the deviation of the actual power transfer of the area $P_{a}(t)$ from its scheduled value $P_{a}^{0}$.

The level of response for a given area is the output of a PI controller fed by the corresponding ACE (1):

$$
R(t)=-\frac{\alpha}{\lambda P_{r t}} \int_{0}^{t} A C E(T) d T-\frac{\beta}{\lambda P_{r t}} A C E(t)
$$

where $\alpha, \beta$ are the integral and proportional gains and $P_{r t}$ is the total positive control range, i.e. the total amount of positive reserves (power that can be generated on top of scheduled production). The total amount of negative reserves is $-P_{r t}$.

The expression of $R(t)$ in Eq. (2) may assume any value. However, AGC parameters $\alpha, \beta$ and $\lambda$ should be tuned as to assure that, for the designed reference incident, the whole amount of reserves $P_{r t}$ is activated. Arbitrarily, one can associate the full activation of reserves with $R(t)= \pm 1$; accordingly, the setpoint of a participating generator $i$ is:

$$
P_{s p}^{i}(t)=P_{0}^{i}+r(t) P_{r}^{i}
$$

where $P_{0}^{i}$ is the scheduled production, $P_{r}^{i}$ is the control range and the limited level of response $r(t)$ is obtained as follows:

$$
\begin{array}{ll}
r(t)=1 & \text { if } R(t) \geq 1 \\
r(t)=-1 & \text { if } R(t) \leq-1 \\
r(t)=R(t) & \text { otherwise }
\end{array}
$$

to take into account cases in which the reserves are exhausted.

The value of $r(t)$ in Eqs. (4 6) is not computed continuously at any instant, but sampled and sent to participating units at intervals of $T_{s}$. The value of $T_{s}$ is in the order of a few seconds. This is to avoid that FRRs react to rapidly varying imbalances, such as those originating from inter-area oscillations. Furthermore, a maximum variation of $r(t)$ over a cycle can be imposed to account for realistic ramp up and ramp down times of the available units.

The participating units start responding after a delay $T_{d}$ that takes into account measurement delays, communication delays and actuation delays. Since frequency can be measured almost instantaneously, it is the computational time to obtain the tie-line flows from the SCADA/EMS that determines the measurement delay. Usually $T_{d}$ is a multiple of $T_{s}$.

The above control scheme can be extended to industrial loads which are capable and willing to modulate their active power use: in the following, this novel control scheme is referred to as Automatic Generation and Load Control (AGLC). In fact, from a system perspective, an increase in active power production from a generator, in response to a positive regulation signal $r$, is equivalent to a decrease in active power consumption from a load and conversely. 
However, a fundamental difference between synchronous machines and industrial loads is that a synchronous machine $i$ is able to continuously vary its production within its control range (between $P_{0}^{i}-P_{r}^{i}$ and $P_{0}^{i}+P_{r}^{i}$ ) and sustain that level of production for an indefinite amount of time. It is unlikely that most industrial loads can do the same because, in addition to provide FRR service, they have to cope with process constraints and avoid any significant disruption to their main industrial use. For instance:

- some loads may not be able to control their consumption in a continuous way (e.g. tap-controlled loads have only a finite amount of tap positions corresponding to different levels of consumption);

- some loads may not be able to offer equal positive and negative reserves (e.g. industrial loads have high utilization levels, so it is more likely that they can offer a decrease in consumption rather than an increase);

- some loads may have some constraints regarding the length of their commitment (e.g. thermostatic loads have to guarantee that the controlled temperature stays within some bounds, metal casting loads have to avoid solidification).

In this respect, the AGLC scheme has to accommodate discrete, unsymmetrical and time-bounded commitments. The individual process constraints of load $j$, as summarised nonexhaustively by the above list, are mapped into five parameters:

- subset of feasible values of response $R_{\text {set }}^{j} \subset[-1 ; 1]$

- maximum positive control range $P_{r+}^{j}$

- maximum negative control range $P_{r-}^{j}$

- maximum energy surplus $E_{r+}^{j}$

- maximum energy deficit $E_{r-}^{j}$

The above terms are consistent with AGC terminology: it is assumed that a reduction of load consumption corresponds to a positive reserve control range, and energy surplus is the energy not consumed by the load. Negative reserve control range and energy deficit are defined conversely.

The setpoint of a participating load $j$ is given by:

$$
\begin{aligned}
P_{s p}^{j}(t) & =P_{0}^{j}-r^{j}(t) P_{r+}^{j} \\
& \text { if } \quad r^{j}(t) \geq 0 \text { and } \int_{0}^{t}\left(P_{s p}^{j}(T)-P_{0}^{j}\right) d T>E_{r-}^{j} \\
P_{s p}^{j}(t) & =P_{0}^{j}+r^{j}(t) P_{r-}^{j} \\
& \text { if } \quad r^{j}(t)<0 \text { and } \int_{0}^{t}\left(P_{s p}^{j}(T)-P_{0}^{j}\right) d T<E_{r+}^{j} \\
P_{s p}^{j}(t) & =P_{0}^{j} \quad \text { otherwise }
\end{aligned}
$$

where $r^{j}(t)$ is intended as the closest value to $r(t)$ belonging to the subset $R_{\text {set }}^{j}$.

Symmetrically, Eq. (3) is modified as follows to allow different positive and negative control ranges also for each participating generator $i$ :

$$
\begin{array}{ll}
P_{s p}^{i}(t)=P_{0}^{i}+r(t) P_{r+}^{i} & \text { if } r(t) \geq 0 \\
P_{s p}^{i}(t)=P_{0}^{i}-r(t) P_{r-}^{i} & \text { if } r(t)<0
\end{array}
$$

For simplicity's sake, it is imposed again that the total amount of positive reserves, from both generators and loads, is $P_{r t}$ and $-P_{r t}$ the total amount of negative reserves.

\section{PROCESS SYSTEM MAPPING EXAMPLES}

Industrial loads that can successfully participate in FRR control are likely to have one or more of the desirable common characteristics which are listed below:

- steady or predictable power consumption;

- low utilization factor;

- continuous variation possibility (or high tap resolution in case of tap-controlled loads);

- high level of process automation;

- low wear and tear impact from increased operation;

- Combined Heat and Power (CHP) or in-plant generation;

- energy storage possibility;

- intermediate product stockpiling possibility;

- high influence of electricity costs on final product;

- experience in provision of RRs (FRRs are in fact faster and automatic RRs).

In the following, four real industrial processes, an aluminium smelter, a Liquefied Natural Gas (LNG) plant, a cement processing plant and a greenhouse are mapped into their 5-parameter descriptions (missing data are replaced by reasonable guesses). The objective of this section is not to provide an accurate analysis of each load, but to show how process flexibility can be described in a simple way to fit the proposed AGLC scheme.

\section{A. Aluminium smelter}

Aluminium smelting is a highly electricity-intensive process. A smelter consists of several pots operated at high DC current. Tap-controlled rectifiers determine the incoming voltage and, thus, the power consumption. Because of the significant thermal inertia, energy can be accumulated, and borrowed, for short amounts of time [8].

The aluminium smelter considered in [8] represents, to the authors' knowledge, the first and only experiment (to date) in provision of FRR from industrial loads. It can provide $\pm 15 \mathrm{MW}$ of regulation around its scheduled consumption of $P_{0}^{A}=550 \mathrm{MW}$ (out of which, $470 \mathrm{MW}$ are for the smelting process alone):

- $P_{r+}^{A}=15 \mathrm{MW}$

- $P_{r-}^{A+}=-15 \mathrm{MW}$

Power consumption is adjusted through a mechanical tap changer whose resolution is $0.75 \mathrm{MW}(0.75 / 15=0.05 \mathrm{pu})$ per step. This leads to:

- $R_{\text {set }}^{A}=\{-1 ;-0.95 ; \ldots ;-0.05 ; 0 ; 0.05 ; \ldots ; 0.95 ; 1\}$

It is assumed that the smelter can borrow a maximum energy surplus equal to its full positive control range for as long as 36 minutes $(0.6 \mathrm{~h})$; let its maximum energy deficit be equal to its full negative control range for as long as $1 \mathrm{~h}$ :

- $E_{r+}^{A}=9 \mathrm{MWh}$

- $E_{r-}^{A+}=-15 \mathrm{MWh}$

This industrial site comprises as well four in-plant generators, for a combined capacity of $732 \mathrm{MW}$, which do not presently provide any ancillary service. Additional flexibility might be offered if those units were allowed to participate in FRR control.

\footnotetext{
${ }^{1}$ The smelter actually provides Regulating Reserve, a service that falls into the broad category of FRR.
} 


\section{B. $L N G$ plant}

LNG plants consume large amounts of electricity to transform natural gas into LNG, which is easier to transport because of its reduced volume. The process consists of three stages: pre-cooling, liquefaction and sub-cooling. Large electric motors are used to drive the corresponding compressors. Compressor load may be temporarily reduced as long as the sub-cooling is sufficient to condense the remaining vapour and the plant operation is stable [10].

The LNG plant considered in [10] is not presently participating in any frequency reserve scheme. The pre-cooling and sub-cooling compressors consume $65 \mathrm{MW}$ each, while the liquefaction compressor $32 \mathrm{MW}$. Given the high utilization factor, it is likely that only positive reserve (corresponding to a $20 \%$ curtailment of pre-cooling and sub-cooling loads) can be committed around the scheduled consumption $P_{0}^{B}=230 \mathrm{MW}$ :

- $P_{r+}^{B}=26 \mathrm{MW}$

- $P_{r-}^{B}=0 \mathrm{MW}$

Since pre-cooling and sub-cooling compressors are driven by variable speed motors, they can vary their consumption continuously within the defined $80-100 \%$ range:

- $R_{\text {set }}^{B}=[0 ; 1]$

It is assumed that the compressors can withhold consumption equal to their full positive control range for as long as 12 minutes $(0.2 \mathrm{~h})$ :

- $E_{r+}^{B}=5.2 \mathrm{MWh}$

- $E_{r-}^{B}=0 \mathrm{MWh}$

If the five in-plant generators, of a combined capacity of $265 \mathrm{MW}$, could act as FRRs, further flexibility might be achieved.

\section{Cement processing plant}

The production of cement requires to crush different stones into a fine powder and blend them in the correct proportions. The process is extremely electricity-intensive. The different rock crushers, however, can be shut down for a short time while production goes on uninterrupted by using the stockpiled material which has been already crushed [11].

The cement processing plant considered in [11] presently takes part in $\mathrm{RR}^{2}$ control, but it could potentially provide more remunerative FRRs. For simplicity's sake, it is supposed that the $22 \mathrm{MW}$ rock crushing consumption at full power is subdivided into 11 rock crushers worth $2 \mathrm{MW}$ each. In normal operation, 7 rock crushers are working at full power, totalling a $14 \mathrm{MW}$ consumption. The scheduled consumption is $P_{0}^{C}=25 \mathrm{MW}$, including $11 \mathrm{MW}$ of other processes. The plant can temporarily operate in a flexible way with as low as 2 crushers or as high as 11:

- $P_{r+}^{C}=10 \mathrm{MW}$
- $P_{r-}^{C}=-8 \mathrm{MW}$

Consumption is not varied in a continuous way, but by merely switching on and off the rock crushers. The 4 crushers standing idle make up negative reserve with a resolution of

\footnotetext{
${ }^{2}$ The cement plant actually participates to the Peak Load Reduction Program, which belongs to the RR class.
}

2 MW ( $1 / 4=0.25 \mathrm{pu})$. Positive reserve (provided by 5 crushers ready to be shed) has a resolution of $2 \mathrm{MW}$ as well $(1 / 5=0.2 \mathrm{pu})$ :

- $R_{\text {set }}^{C}=\{-1 ;-0.75 ;-0.5 ;-0.25 ; 0 ; 0.2 ; 0.4 ; 0.6 ; 0.8 ; 1\}$

In this case, rather than the energy which is absorbed and borrowed by the process, it is the amount of stockpiled material that determines the possible length of commitment. However, supposing that in normal operation the stockpile is kept at half capacity, and that it takes 4 crushers for 1 hour to crush this amount of rocks, equivalent energy surplus and deficit can be found:

- $E_{r+}^{C}=8 \mathrm{MWh}$

- $E_{r-}^{C}=-8 \mathrm{MWh}$

Along with the crushing process, cement production requires as well large amounts of heat for the drying process, often produced with CHP units which could take part in FRR control. However, this plant does not include any on-site CHP generator.

\section{Greenhouse}

The following example shows that it is not only heavy industry that can benefit from FRR provision, but also other industrial processes may be fit for this service. Greenhouses need to supply heat, $\mathrm{CO}_{2}$ and light in order to grow crops. The natural process of plant growing is not affected by occasional disturbances. The supply of heat, $\mathrm{CO}_{2}$ and light can thus be temporarily interrupted without consequences [12].

The greenhouse considered in [12] presently provides RR 3 but hereafter we analyse its potential to act as a FRR. It is assumed that the scheduled consumption of this plant is $P_{0}^{D}=$ $10 \mathrm{MW}$, including 7.5 MW of lighting load, which can be freely dimmed between 3 and $12 \mathrm{MW}$, or completely switched off:

- $P_{r+}^{D}=7.5 \mathrm{MW}$

- $P_{r-}^{D}=-4.5 \mathrm{MW}$

When negative reserves are needed, lighting load can be increased continuously between 7.5 and 12 MW. Positive reserves can be obtained from lighting load with a complete switch off of $7.5 \mathrm{MW}$ (1 pu), or continuously from 7.5 to $3 \mathrm{MW}$, for a total of $4.5 \mathrm{MW}(4.5 / 7.5=0.6 \mathrm{pu})$ :

- $R_{\text {set }}^{D}=[-1 ; 0.6] \cup\{1\}$

Since what counts is that the crops receive roughly 15 hours per day of full light (at $12 \mathrm{MW}$ ), which is equivalent to the scheduled load of 7.5 MW over the whole day, the greenhouse is capable of both positive and negative FRR regulation for rather long periods of time. Supposing that the crops can withstand for 1 hour, without any effect on their growing, both full power lighting and no lighting, it holds:

- $E_{r+}^{D}=7.5 \mathrm{MWh}$

- $E_{r-}^{D}=-4.5 \mathrm{MWh}$

Additionally, the greenhouse comprises two on-site CHP units, totalling $6.6 \mathrm{MW}$ of capacity, which are used to provide heat and $\mathrm{CO}_{2}$ to the crops and to power the lights when in use. Participation of these units to FRR control could further enlarge the amount of provided reserves.

\footnotetext{
${ }^{3}$ The greenhouse actually provides Short Term Operating Reserve to the National Grid in the UK. This service can be classified as RR.
} 


\section{FREQUENCY CONTROL IN THE NORDIC SYSTEM}

The synchronously interconnected systems of East Denmark, Finland, Norway and Sweden constitute the Nordic system. The full line in Fig. 1 1 shows the wind power installed capacity in the Nordic system [13]: this dramatic increase makes the Nordic system a compelling case to analyse the impact of renewable energy penetration. The challenges met by the Nordic TSOs are representative of those met by other operators in the UK, US and continental Europe, as they strive to meet ambitious sustainability goals.

Figure 1 shows also a measure of the degrading frequency quality in the Nordic system: the number of frequency incidents (i.e. minutes per month during which system frequency lies outside the $49.9-50.1 \mathrm{~Hz}$ interval) is plotted in dashed line [14]. Investigating the correlation between frequency quality and renewable energy penetration is outside the scope of this paper. Although other phenomena of market origin are also responsible [15], the figure suggests a correlation between frequency deviations and renewable generation.

As of 2012, the Nordic system frequency control is based on one FCR service, Normal Operation Reserve (NOR), and two RR services, Fast and Slow Active Disturbance Reserve (FADR, SADR); an emergency FCR scheme, Disturbance Reserve (DR), is activated when the frequency falls below $49.9 \mathrm{~Hz}$. Table I] reviews the above mentioned services [16].

Concerned with the increase of frequency incidents, the Nordic TSOs have agreed on the introduction of a new FRR service. In December 2012, the Norwegian operator Statnett became the first Nordic TSO to operate FRRs [17]: the other operators will introduce similar services by early 2013 .

The FRR implementation in the Nordic system does not include the term $\Delta P_{a}(t)$ in the ACE definition (1), as maintaining the planned flows is not one of the objectives of this service [14]. The ACE of the Nordic system is thus merely the frequency deviation $\Delta f(t)$. Equation (2), representing the level of response, is thus replaced by the following:

$$
R(t)=-\frac{\alpha}{P_{r t}} \int_{0}^{t} \Delta f(T) d T-\frac{\beta}{P_{r t}} \Delta f(t)
$$

In comparison with other FRR services, the Nordic FRR control is expected to show shorter measurement delays as only the frequency is considered in the ACE.

Although loads are excluded from provision of NORs, as of 2007 more than one third of the remaining reserves were provided by industrial processes in the Nordic system, mainly aluminium smelters, metal processing and the forestry, pulp and paper industry [7]. Participation of loads as FRRs is yet unspecified, as the current version of the Nordic Grid Code (2007) does not include FRR control at all [16].

TABLE I

FREQUENCY CONTROL SERVICES IN THE NORDIC SYSTEM

\begin{tabular}{|c|c|c|c|c|c|}
\hline Service Name & NOR & DR & FRR & FADR & SADR \\
\hline \hline Service Type & FCR & Emergency & FRR & RR \\
\hline Action Type & \multicolumn{3}{|c|}{ Automatic } & \multicolumn{2}{|c|}{ Manual } \\
\hline Hierarchy & \multicolumn{3}{|c|}{ Local } & \multicolumn{3}{|c|}{ Centralised } \\
\hline Response [min] & $<2$ & $<0.5$ & $<5$ & $<15 \quad \geq 15$ \\
\hline Loads (as of 2007) & No & Yes & - & Yes \\
\hline
\end{tabular}

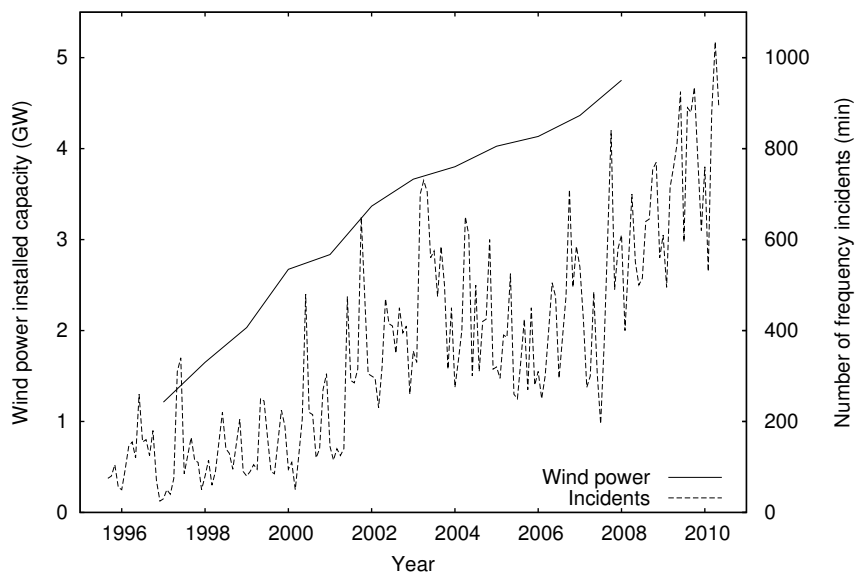

Fig. 1. Nordic system trends: wind power installed capacity and number of frequency incidents, i.e. minutes per month during which system frequency lies outside the $49.9-50.1 \mathrm{~Hz}$ interval (from [13] and [14], respectively).

\section{DyNAMiC SIMULATION OF A POWER IMBALANCE}

Long-term dynamic simulations have been performed using RAMSES4 software on a modified version of the so-called Nordic32 test system, proposed by K. Walve (at that time with Svenska Kraftnät, the Swedish TSO) and detailed in [20]. As indicated in this reference, the system is fictitious but similar to the Swedish system and to the Nordic interconnection as a whole (at the time of setting up this test system).

The system includes 52 (generator and transmission) buses and 80 branches. When including the distribution buses and transformers, there are a total of 74 buses and 102 branches, respectively. The synchronous machines are equipped with generic excitation systems, voltage regulator, power system stabilizers, speed governors and turbine models. Loads are represented by the restorative load model described in [19] and intermittent generators as power injections.

FCR (NOR) control is performed by the speed governors of the hydro generators. Thermal units do not act as FCRs.

Three scenarios have been considered:

1) base case;

2) same as 1, with AGC involving some hydro generators;

3) same as 1, with AGLC involving some industrial loads providing $50 \%$ of FRRs along with some hydro generators providing the remaining amount.

The disturbance considered is an imbalance with a ramp of $10 \mathrm{MW} / \mathrm{s}$, from $t=10 \mathrm{~s}$ to $t=20 \mathrm{~s}$, for a total of $100 \mathrm{MW}$. The sudden drop in frequency observable in Fig. 2 is the system response to the power imbalance. After a few seconds, however, FCRs start to act and stabilise the frequency drop. In the base case nothing happens from then on, and frequency is not restored, since there is no FRR control. DRs do not act because the $49.9 \mathrm{~Hz}$ threshold is not reached, while the action of RRs (FADRs and SADRs) is not considered in these simulations as they would normally be activated only at a later stage to relieve units participating in FCR and FRR controls.

\footnotetext{
${ }^{4}$ Acronym for Relaxable Accuracy Multithreaded Simulator of Electric power Systems, developed in the course of the research reported in [18], [19].
} 


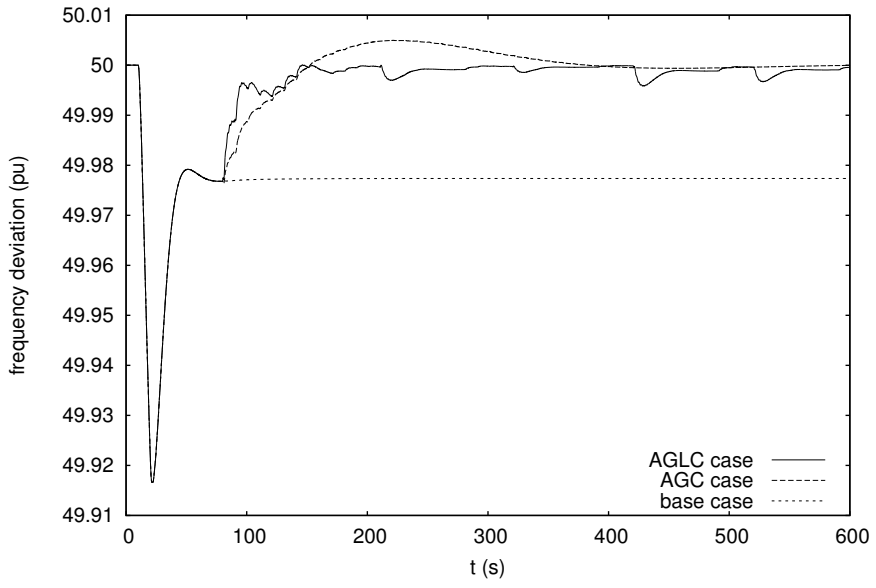

Fig. 2. Comparison of frequency deviation in different simulated scenarios.

In both cases where FRR control is present, after around one minute the FRRs start to act to restore system frequency. In the AGC case, the frequency slightly overshoots and then recovers. In the AGLC case, some ripples are observed later in the simulation in correspondence with participating loads hitting their maximum energy surplus and thus being relieved from participation to FRR service. Ultimately, the frequency restores to its nominal value.

This is an academic case in which the power imbalance occurs as a clear-cut disturbance at the beginning of the simulation, when the system is otherwise in perfect equilibrium. In reality, both load consumption and renewable generation are always changing, and the FCR, FRR and RR controls are almost always in action. However, the simulated imbalance can be seen as a worst-case scenario corresponding to increasing load or wind generators ramping down steeply.

The purpose of this simulation is to show that FRRs can be provided in an equally satisfactory way by the conventional generators solely or by a combination of both conventional generators and flexible industrial processes.

\section{CONCLUSION}

The power industry is facing significant challenges in order to meet its ambitious sustainability goals. A troublesome increase in the number of frequency incidents should ignite the interest in frequency control: indeed, regardless of the specific causes, any frequency incident can be attributed to insufficient reserves. A more active role of industrial processes in power system operation is envisaged in order to compensate for the increased intermittent generation. Advances in industrial automation are paving the way towards this objective.

In this paper, a FRR control scheme with participation of industrial loads is proposed. The AGC scheme is revisited and extended in order to accommodate load participation, described by as few as five parameters in the novel AGLC scheme proposed. Four representative industrial processes are reviewed, and their flexibility mapped with the proposed 5parameter description. Simulations performed on a 74-bus test system confirm that loads can be successfully integrated as FRRs. This result enlarges the pool of available reserves.
The implementation of AGLC schemes in real systems and the economic aspects of FRR service provision should be further investigated on a system-by-system basis to assure that the various technical requirements are met. Appropriate incentives are necessary to encourage the exploitation of industrial loads as FRRs. This might also sprout interest in revisiting the decades-old frequency control services in light of increased load participation and renewable penetration.

Further investigations might be also directed towards the provision of voltage control services, possibly enabled by industrial loads interfaced through power electronic converters and capable of controlling their reactive consumption.

\section{REFERENCES}

[1] REAL-SMART project. http://www3.imperial.ac.uk/realsmart, accessed February 2013.

[2] Y. G. Rebours, D. S. Kirschen, M. Trotignon, and S. Rossignol, "A survey of frequency and voltage control ancillary services - Part I: technical features," IEEE Trans. Power Syst., vol. 22, pp. 350-357, Feb. 2007.

[3] D. S. Callaway and I. A. Hiskens, "Achieving controllability of electric loads," Proceedings of the IEEE, vol. 99, pp. 184-199, Jan. 2011.

[4] B. J. Kirby, "Load response fundamentally matches power system reliability requirements," in Proc. 2007 IEEE PES General Meeting, pp. 1-6, June 2007.

[5] T. Samad and S. Kiliccote, "Smart grid technologies and applications for the industrial sector," Computers \& Chemical Engineering, vol. 47, pp. 76-84, Dec. 2012.

[6] Operational Reserve Ad Hoc Team Report. v. 6, ENTSO-E, May 2012.

[7] G. Heffner, C. Goldman, B. Kirby, and M. Kintner-Meyer, Loads Providing Ancillary Services: Review of International Experience. LBNL62701, Lawrence Berkeley National Laboratory, May 2007.

[8] D. Todd, M. Caufield, B. Helms, , M. Starke, B. Kirby, and J. Kueck, Providing Reliability Services through Demand Response: A Preliminary Evaluation of the Demand Response Capabilities of Alcoa Inc. ORNL/TM-2008/233, Oak Ridge National Laboratory, Jan. 2009.

[9] P. Kundur, Power system stability and control. The EPRI power system engineering series, McGraw-Hill, 1994.

[10] S. Vist, M. Svenning, H. F. Valle, H. Ormbostad, G. B. Gabrielsen, D. Pedersen, R. I. Nielsen, J. Pettersen, and A. O. Fredheim, "Start-up experiences from Hammerfest LNG, a frontier project in the north of Europe," in Proc. 16th Liquefied Natural Gas Conference and Exhibition (LNG16), pp. 1-16, Apr. 2010.

[11] G. Epstein, M. D'Antonio, C. Schmidt, J. Seryak, and C. Smith, "Demand response enabling technologies and approaches for industrial facilities," in Proc. 27th Industrial Energy Technology Conference (IETC), pp. 1-7, May 2005.

[12] Greenhouse greens the grid (from Flexitricity Archive, Aug. 2011). http://www.flexitricity.com/file/Kaaij_Flexitricity_Case_Study_1_0.pdf, accessed February 2013.

[13] ENTSO-E Annual Statistics. https://www.entsoe.eu/publications/formerassociations/nordel/annual-statistics/, accessed February 2013.

[14] D. Whitley and O. Gjerde, "LFC/AGC - Nordic and European perspective," in Exchange of balancing services between the Nordic and the Central European synchronous systems, pp. 1-13, Jan. 2011.

[15] Deterministic frequency deviations - root causes and proposals for potential solutions. D/2011/12.105/55 Joint EURELECTRIC - ENTSOE response paper, Dec. 2011.

[16] Nordic Grid Code 2007 (Nordic collection of rules). Jan. 2007.

[17] Oppstart av nytt marked for automatiske sekundarreserver (from Statnett News Archive, Nov. 2012). http://www.statnett.no/no/Nyheterog-media/Nyhetsarkiv/Nyhetsarkiv-2012/Oppstart-av-nytt-marked-forautomatiske-sekundarreserver-/, accessed February 2013.

[18] D. Fabozzi, A. S. Chieh, B. Haut, and T. Van Cutsem, "Accelerated and localized Newton schemes for faster dynamic simulation of large power systems," submitted to IEEE Trans. Power Syst., 2013.

[19] D. Fabozzi, Decomposition, Localization and Time-Averaging Approaches in Large-Scale Power System Dynamic Simulation. PhD thesis, Université de Liège, http://orbi.ulg.ac.be/handle/2268/126720, July 2012.

[20] M. Stubbe, "Long-Term Dynamics - Phase II," Report of CIGRE Task Force 38.02.08, Jan. 1995. 